\title{
Specify Other Preoperative IV Antiemetics Administered
}

National Cancer Institute

\section{Source}

National Cancer Institute. Specify Other Preoperative IV Antiemetics Administered. NCI

Thesaurus. Code C162675.

A request to specify intravenous antiemetics that were administered preoperatively but not included on a list. 